\title{
A Successfully Resected Case of Recurrent Lung and Liver Metastases of Rectal Cancer Treated with XELIRI + Bevacizumab Therapy
}

\author{
Naoya Aisu Yoichiro Yoshida Fuminori Ishii Toru Miyake \\ Shu Tanimura Yoshito Wada Yasushi Yamauchi Seiichiro Hoshino \\ Tomoaki Noritomi Yuichi Yamashita \\ Department of Gastroenterological Surgery, Fukuoka University Graduate School of \\ Medicine, Fukuoka, Japan
}

\section{Key Words}

Colorectal cancer · Liver metastasis · XELIRI

\begin{abstract}
It has been reported that many colorectal cancer (CRC) patients with synchronous or metachronous liver metastases underwent surgery subsequent to neoadjuvant combination chemotherapy with folinic acid, fluorouracil, and oxaliplatin (FOLFOX), folinic acid, fluorouracil, and irinotecan (FOLFIRI), or capecitabine and oxaliplatin (XELOX). However, there are very few reports of the use of capecitabine and irinotecan (XELIRI). We herein report a successfully resected case of recurrent lung and liver metastases of rectal cancer treated with combination chemotherapy with XELIRI + bevacizumab (BV) therapy. A 63-year-old male developed recurrence of a solitary nodule in the right lower lobe of the lung and multiple liver metastases after low anterior resection for rectal cancer 1 year previously. Partial resection of the right lower lobe of the lung was performed and treatment with XELIRI + BV was initiated. A computed tomography scan revealed a reduction in tumor size without any new lesions after four cycles of XELIRI + BV therapy. Partial hepatectomy of S1, S5, and S7 was safely performed. The patient is now undergoing adjuvant chemotherapy and has been free from recurrence for 18 months following surgery. There are only few studies with relatively low patient numbers reporting on the outcome after resection of both pulmonary and hepatic metastases of CRC. We therefore report a patient who underwent sequential resection of pulmonary and hepatic metastases with XELIRI + BV therapy.
\end{abstract}

7-45-1 Nanakuma, Jonan-ku

Fukuoka 814-0180 (Japan)

E-Mail ice13man1978@yahoo.co.jp 


\section{Introduction}

Advancements in systemic chemotherapy for colorectal cancer (CRC) have improved the clinical response rate and expanded the indications for resection to cases that were previously not operable at the initial visit. In addition, the prognosis of patients who undergo radical surgery for metastasis is clearly better than that of patients with inoperable tumors [1].

Several studies have demonstrated the efficacy and safety of capecitabine and irinotecan (XELIRI) therapy as a first-line regimen [2-8]. In the FNCLCC ACCORD 13/0503 study, a randomized, non-comparative study, XELIRI + bevacizumab (BV) and folinic acid, fluorouracil, and irinotecan (FOLFIRI) + BV were found to be similarly effective treatments as chemotherapy alone for metastatic CRC (MCRC) patients with manageable toxicity profiles [2].

Phase II studies have shown that XELIRI therapy is active in mCRC with unresectable liver-only metastases [3]. There are, however, no reports about the use of the XELIRI + BV regimen in the neoadjuvant treatment for patients with lung and liver metastases of CRC.

\section{Case Report}

A 63-year-old male presented with recurring metastatic rectal cancer in February 2010. The patient had previously undergone low anterior resection for UICC stage II A (T3NOM0) rectal cancer and received tegafur and uracil at a dose of $500 \mathrm{mg} /$ day for half a year. The carcinoembryonic antigen level was $217.8 \mathrm{ng} / \mathrm{ml}$. Tumor recurrence was confirmed on a computed tomography (CT) scan that showed a solitary nodule in the right lower lobe of the lung and multiple liver metastases (in S1, S5, and S7) in February 2011 (fig. 1). Partial resection of the right lower lobe was performed in March 2011. The patient was treated with XELIRI + BV [BV $(7.5 \mathrm{mg} / \mathrm{kg})$, CPT- $11\left(200 \mathrm{mg} / \mathrm{m}^{2}\right)$ on day 1 plus capecitabine $(1,000$ $\mathrm{mg} / \mathrm{m}^{2}$ ) twice daily on days $1-14$, every 3 weeks] after surgery. The carcinoembryonic antigen level decreased to normal, and a CT scan revealed a partial response after four cycles of chemotherapy (fig. 2). Partial resection of S1, S5, and S7 of the liver was then safely performed in August 2011 (fig. 3). No postoperative complications were observed.

The patient underwent postoperative adjuvant therapy consisting of XELIRI + BV for four cycles and Xeloda (capecitabine) + BV for five cycles. He has been free from recurrence for 18 months following surgery.

\section{Discussion}

The treatment of mCRC has evolved significantly over the past 10 years. In particular, combination chemotherapy has become the standard treatment for MCRC, often combined with a biological agent. Such therapies have significantly improved clinical efficacy related to overall response rates, time to tumor progression, and median overall survival. Several studies have reported comparable results between XELIRI and FOLFIRI \pm BV as first-line treatment for mCRC. In the BICC-C randomized trial, patients treated with FOLFIRI exhibited a significantly longer progression-free survival (PFS) compared to those treated with XELIRI [4]. In contrast, in the FNCLCC ACCORD 13/0503 study, a randomized, non-comparative study, the median PFS was 9.3 months in the XELIRI + BV and 9.0 months in the FOLFIRI + BV arms [2]. In the Hellenic Cooperative Oncology Group phase III trial using collateral 
biomarkers, the median PFS was 10.2 months in the XELIRI + BV and 10.8 months in the FOLFIRI + BV arms [5]. These findings suggest that the XELIRI $\pm \mathrm{BV}$ regimen for $\mathrm{mCRC}$ is effective and equivalent to the FOLFIRI \pm BV regimen.

In the BICC-C trial, the incidence of the most prominent grade 3-4 adverse events with XELIRI were the following: diarrhea (47\%), neutropenia (32\%), dehydration (19\%), nausea $(18 \%)$, and vomiting (6\%). Twenty-five percent of the patients discontinued the regimen and the treatment was prematurely terminated [4].

In the CAIRO trial, patients assigned to the XELIRI (combination) arm presented more frequently with diarrhea (27\%), nausea/vomiting (9\%), neutropenia (7\%), febrile neutropenia (7\%), and hand-foot syndrome (7\%) as grade 3-4 toxicities [6]. In the EORTC 40015 study, patients treated with XELIRI demonstrated high rates of grade 3-4 toxicities: diarrhea $(37 \%)$, neutropenia $(14 \%)$, vomiting $(7 \%)$, and nausea $(4 \%)$. Fifty-three percent of the patients required dose reduction and $14 \%$ succumbed to toxicity [7].

In these three trials, the dose of irinotecan was $250 \mathrm{mg} / \mathrm{m}^{2}$. However, in the FNCLCC ACCORD 13/0503 study, the dose of irinotecan was $200 \mathrm{mg} / \mathrm{m}^{2}$ and the incidence of the most prominent grade 3-4 adverse events with XELIRI were lower: diarrhea (12\%), neutropenia (18\%), general fatigue (14\%), nausea (3\%), and vomiting (7\%). Only $8 \%$ of the patients discontinued the regimen, and the treatment was prematurely terminated. In our case, we administered the same regimen, and the patient developed only grade 2 hand-foot syndrome.

Neoadjuvant chemotherapy for CRC with resectable liver metastasis results in a reduction in tumor size and a safe tumor margin. Moreover, Adam et al. [1] mention that the 5year survival rate of cases in which resection became possible following preoperative chemotherapy is the same as the resection results of cases in which resection was possible at the time of diagnosis. As the recurrence rate following radical resection is high, in our department the basic policy is to carry out a four-course capecitabine and oxaliplatin $(\mathrm{XELOX})+\mathrm{BV}$ or XELIRI + BV treatment regarding preoperative chemotherapy against all colon cancer liver metastasis cases, regardless of whether it is resectable or unresectable, and then resecting it following re-evaluation if possible.

There is no unified view on which of the XELOX and XELIRI regimens should be given priority. According to a report by Yoshida et al. [8], approximately $20 \%$ of all colon cancer cases exhibit oxaliplatin resistance. Therefore, in such cases, the irinotecan regimen may also be the first-line regimen, thereby prolonging the prognosis. In the cases treated at our department, preoperative four-course XELIRI + BV therapy was carried out as a FUTURE1001 trial via the Institutional Review Board (trial registration: UMIN000005060), and then a radical resection was safely carried out.

\section{Conclusion}

The promising results presented in this paper suggest that the combination of XELIRI + $\mathrm{BV}$ may represent a novel, highly active and advantageous regimen for patients with mCRC.

\section{References}

1 Adam R, Wicherts DA, de Haas RJ, et al: Patients with initially unresectable colorectal liver metastases: is there a possibility of cure? J Clin Oncol 2009;27:1829-1835. 


\section{Case Reports in Oncology}

\begin{tabular}{|c|c|}
\hline \multicolumn{2}{|c|}{ Case Rep Oncol 2013;6:143-147 } \\
\hline DOI: $\underline{10.1159 / 000349973}$ & $\begin{array}{l}\text { (c) } 2013 \text { S. Karger AG, Base } \\
\text { www.karger.com/cro }\end{array}$ \\
\hline
\end{tabular}

Aisu et al.: A Successfully Resected Case of Recurrent Lung and Liver Metastases of Rectal Cancer Treated with XELIRI + Bevacizumab Therapy

2 Ducreux M, Adenis A, Mendiboure J, et al: Efficacy and safety of bevacizumab (BEV)-based combination regimens in patients with metastatic colorectal cancer (mCRC): randomized phase II study of BEV + FOLFIRI versus BEV + XELIRI (FNCLCC ACCORD 13/0503 study). J Clin Oncol 2009;27:4086.

-3 Skof E, Rebersek M, Hlebanja Z, Ocvirk J: Capecitabine plus Irinotecan (XELIRI regimen) compared to 5FU/LV plus Irinotecan (FOLFIRI regimen) as neoadjuvant treatment for patients with unresectable liveronly metastases of metastatic colorectal cancer: a randomised prospective phase II trial. BMC Cancer 2009;9:120.

4 Fuchs CS, Marshall J, Mitchell E, et al: Randomized, controlled trial of irinotecan plus infusional, bolus, or oral fluoropyrimidines in first-line treatment of metastatic colorectal cancer: results from the BICC-C Study. J Clin Oncol 2007;25:4779-4786.

5 Pectasides D, Papaxoinis G, Kalogeras KT, et al: XELIRI-bevacizumab versus FOLFIRI-bevacizumab as firstline treatment in patients with metastatic colorectal cancer: a Hellenic Cooperative Oncology Group phase III trial with collateral biomarker analysis. BMC Cancer 2012;12:271.

6 Koopman M, Antonini NF, Douma J, et al: Sequential versus combination chemotherapy with capecitabine, irinotecan, and oxaliplatin in advanced colorectal cancer (CAIRO): a phase III randomised controlled trial. Lancet 2007;370:135-142.

7 Köhne CH, De Greve J, Hartmann JT, et al: Irinotecan combined with infusional 5-fluorouracil/folinic acid or capecitabine plus celecoxib or placebo in the first-line treatment of patients with metastatic colorectal cancer. EORTC study 40015. Ann Oncol 2008;19:920-926.

-8 Yoshida Y, Hasegawa J, Nezu R, et al: Clinical usefulness of mitochondrial transcription factor A expression as a predictive marker in colorectal cancer patients treated with FOLFOX. Cancer Sci 2011;102:578-582.

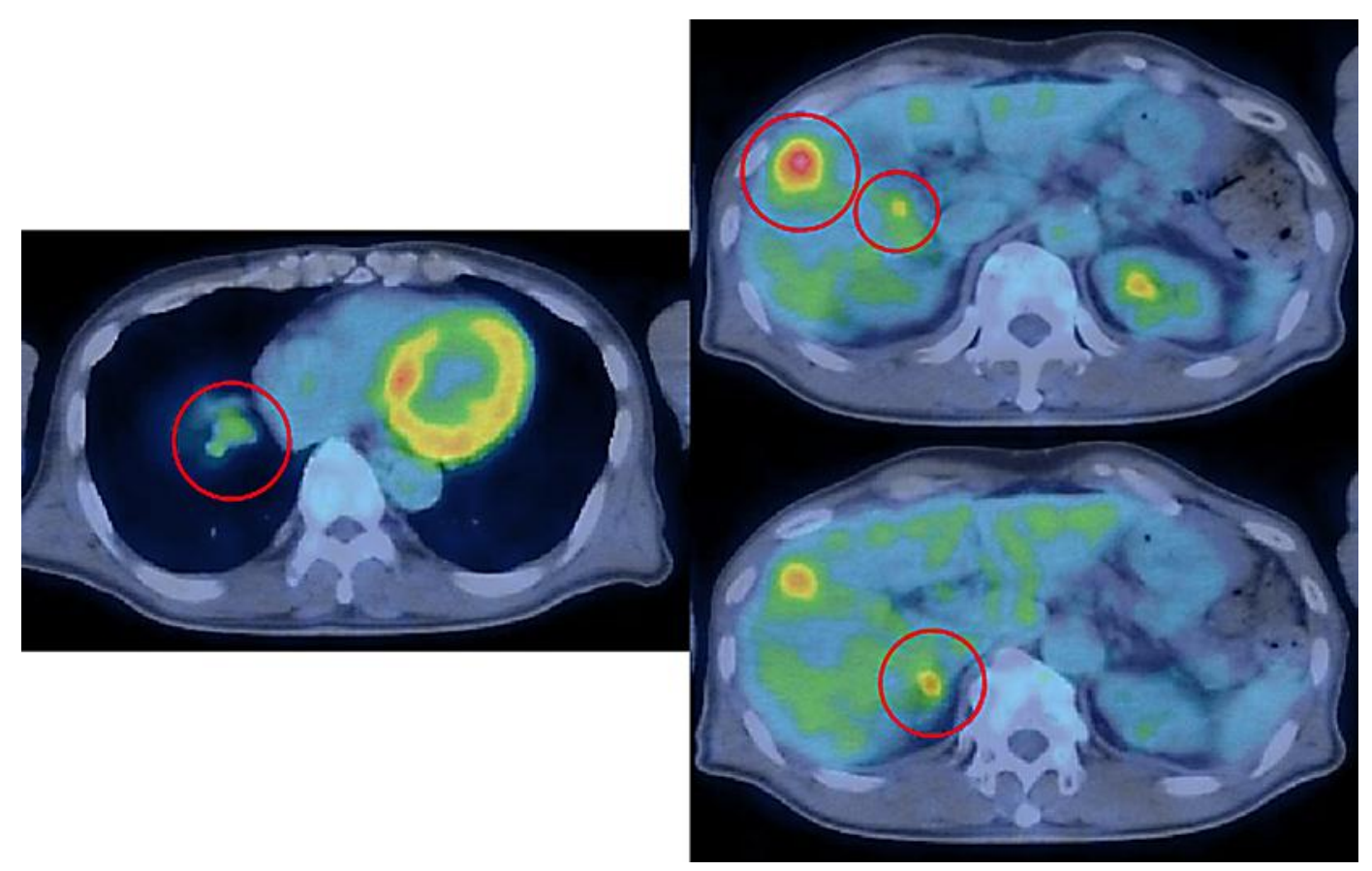

Fig. 1. Positron emission tomography/CT revealed high-grade accumulation of $\left[{ }_{18} \mathrm{~F}\right]$-fluordeoxyglucose in a solitary nodule of the right lower lobe of the lung and in three nodules in segments 1, 5, and 7 of the liver. 


\section{Case Reports in Oncology}

Case Rep Oncol 2013;6:143-147

DOI: $\underline{10.1159 / 000349973}$

Aisu et al.: A Successfully Resected Case of Recurrent Lung and Liver Metastases of

Rectal Cancer Treated with XELIRI + Bevacizumab Therapy

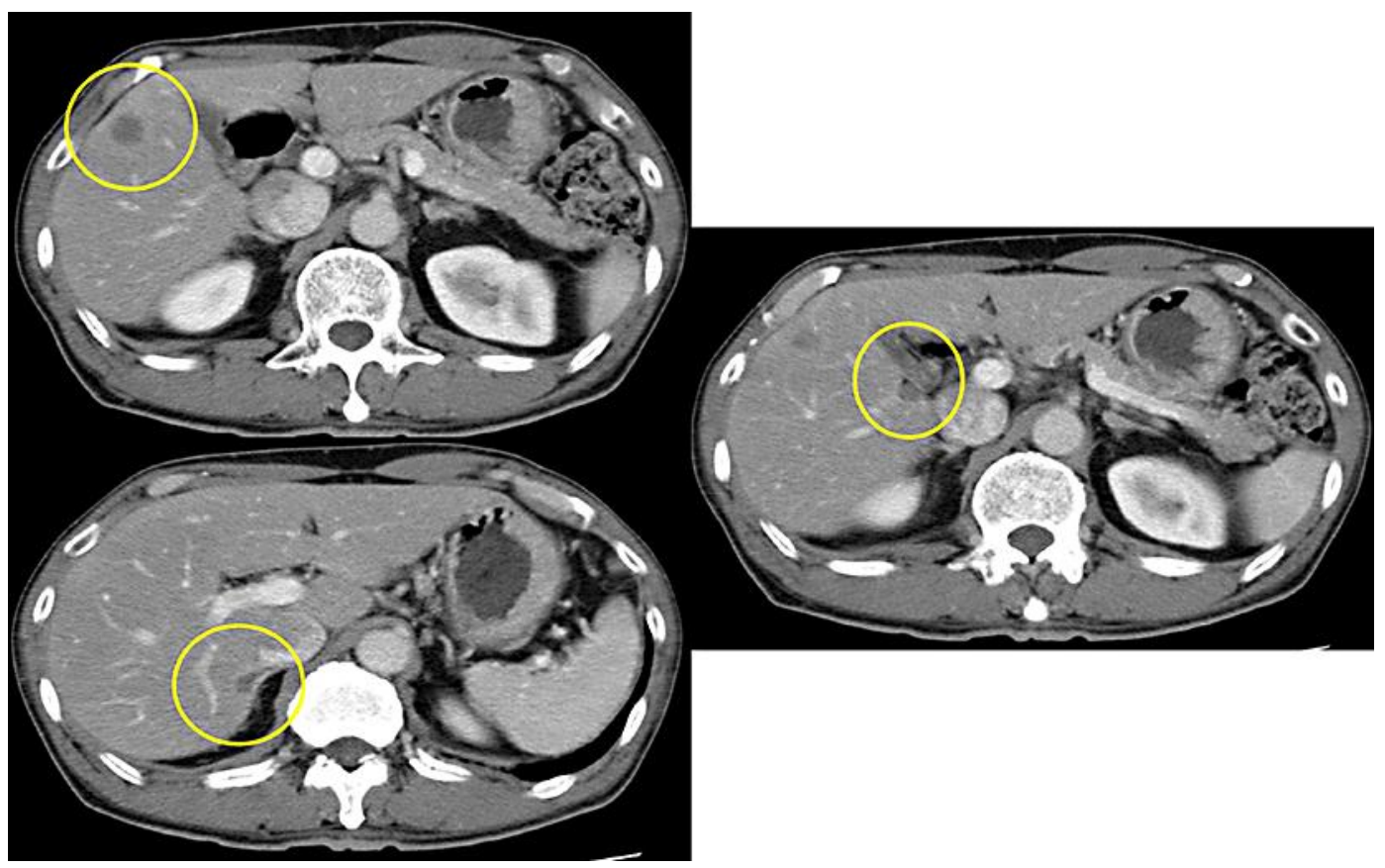

Fig. 2. Enhanced CT showed partial response.
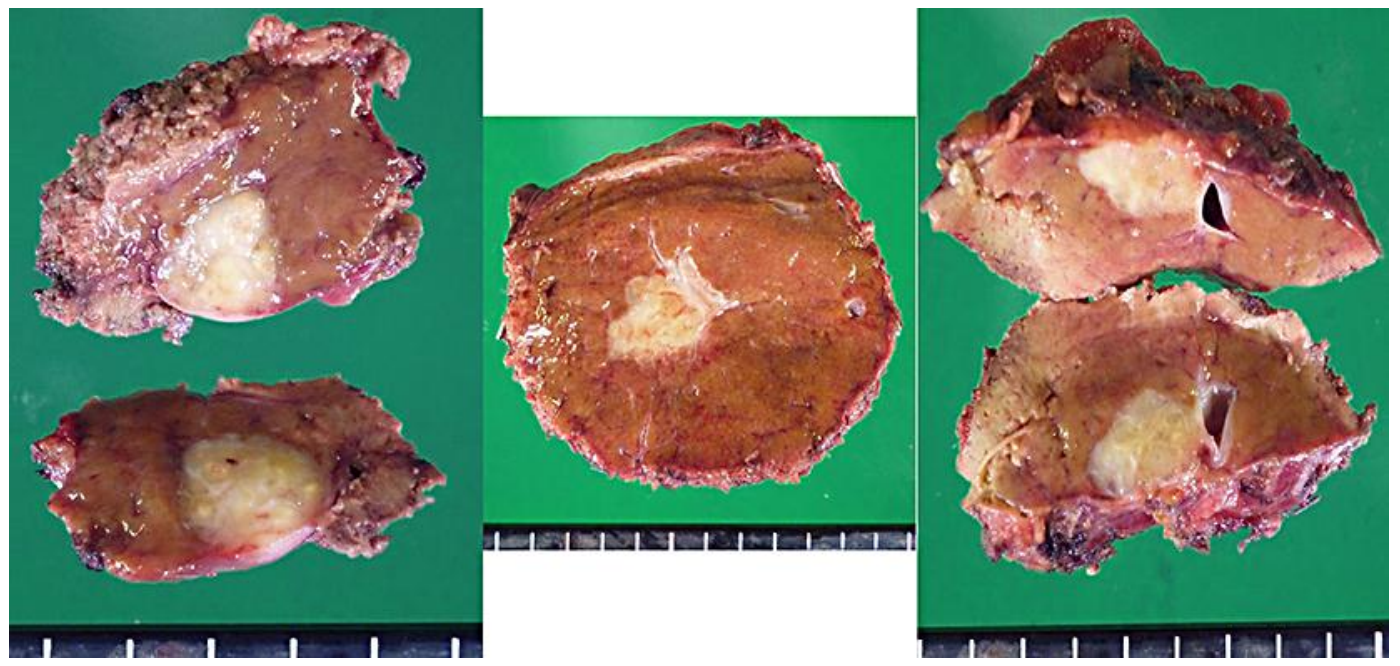

Fig. 3. A resected specimen showed a 10-, 17-, and 10-mm tumor in segments 1, 5, and 7, respectively. 